논문 2019-1-8 http://dx.doi.org/10.29056/jsav.2019.06.08

\title{
$\mathrm{DFB}$ 레이저의 빔 분포 시뮬레이션과 검정
}

\author{
권기영*, 기장근*
}

\section{Simulation and Examination for Beam Profile of DFB Laser}

\author{
Kee-Young Kwon*, Jang-Geun Ki*
}

요 약

광대역 광통신 시스템에 사용되는 레이저는 우수한 주파수 선택성과 모드 안정성을 가져야한다. $\mathrm{DFB}$ (Distributed Feedback) 레이저는 고주파로 전류 변조를 하더라도 발진 주파수의 변화가 적다.

본 연구에서는 무반사 코팅을 하지 않은, 두 거울 면을 가진 $1.55 \mathrm{um}$ 의 파장을 갖는 $\mathrm{DFB}$ 레이저에서 이득 격자와 굴절률 격자가 동시에 존재할 때, 시뮬레이션 소프트웨어를 개발하여 종 방향으로의 발진 모드의 빔 분포를 해석하였다. 굴절률 격자와 이득 격자가 거울 면에서 갖는 위상 값의 변화에 따라서 $\mathrm{DFB}$ 레이저의 빌진 모드에 대한 빔 분포 $|R(z)|$ 와 $|S(z)|$, 그리고 방사전력비 $P_{l} / P_{r}$ 를 비교 김증하였다. 거울 면에 서의 격자 위상에 관계없이 발진 모드의 문턱 전류를 낮추고 주파수 안정성을 높이기 위해서는, $\kappa L$ 이 8 보다 커야한다.

\section{Abstract}

Lasers for optical broadband communication systems should have excellent frequency selectivity and modal stability. DFB lasers have low lasing frequency shift during high speed current modulation.

In this paper, we have developed a simulation software and analysed beam profiles of a lasing mode in longitudinal direction of an 1.55um DFB laser with two mirrors and without anti-reflection coatings, that have both an index- and gain-gratings. As the phases of the index and gain gratings on the mirror faces are varied, the beam profiles $|R(z)|$ and $|S(z)|$ of the lasing mode with the emitted power ratio $P_{l} / P_{r}$ are analysed and examined. In order to reduce the threshold current of a lasing mode and enhance the frequency stability, $\kappa L$ should be greater than 8 , regardless of the grating phases on the mirror faces.

한글키워드 : $\mathrm{DFB}$ 레이저, 빔 분포, 격자의 위상, 굴절률 격자, 이득 격자

keywords : DFB laser, beam profile, grating phase, index grating, gain grating

\section{1. 서 론}

* 공주대학교 전기전자제어공학부 (email: kky@kongju.ac.kr) 접수일자: 2019.05.31. 심사완료: 2019.06.13. 게재확정: 2019.06.20.
광대역 광통신 시스템에 사용되는 레이저 광 원은 주파수 선택성이 우수하고 안정된 주파수 동작을 하면서, 고주파로 전류 변조를 하더라도 발진 파장의 변화가 적은 $\mathrm{DFB}$ (Distributed Feedback) 레이저가 각광을 받고 있다[1-5]. 
$\mathrm{DFB}$ 레이저는 브래그(Bragg) 산란을 이용하고 있는데, 굴절률 결합 $\mathrm{DFB}$ 레이저의 경우 발진 파장의 축퇴와 거울면의 반사율, 그리고 거울면 의 위상에 따라 발진특성이 민감한 것이 문제이 다. 이러한 문제를 개선하기 위해서 반사 방지 코팅을 한 4 분의 1 파장 위상 천이된 $\mathrm{DFB}$ 레이 저가 제안되었는데, 낮은 잔류 반사율 $(\ll 0.005)$ 이 요구되고 이득의 비선형성을 유발하는 공간적 인 홀 버닝(spatial hole burning) 현상이 심한 것 이 문제이다[6-10].

본 논문에서는 무반사 코팅을 하지 않은, 두 거울 면을 가진 $1.55 \mathrm{um}$ 의 파장을 갖는 $\mathrm{DFB}$ 레 이저에서 이득 격자와 굴절률 격자가 동시에 존 재할 때, 이득 격자와 굴절률 격자 사이의 구조 와 배치 방법에 따라 발진 주파수와 발진 이득, 장축 방향으로의 빔 분포 특성을 이론적으로 해 석한다. 2장에서는 이론적인 바탕을 설명하고, 3 장에서는 굴절률 격자와 이득 격자가 거울 면에 서 갖는 위상 값의 변화에 따라서 $\mathrm{DFB}$ 레이저의 발진 모드에 대한 빔의 분포 $|R(z)|$ 와 $|S(z)|$, 그리고 방사전력비 $P_{l} / P_{r}$ 를 구했다.

\section{2. 시뮬레이션의 이론적 배경}

\section{1 발진 모드와 발진 이득}

굴절률 격자와 이득 격자가 주는 효과를 모두 고려하기 위하여, 굴절률 격자와 이득 격자를 다 음 식과 같이 표현한다.

$$
\begin{aligned}
& n(z)=n+(\Delta n) \cos \left(\frac{2 \pi z}{\Lambda}+\Omega\right) \\
& \alpha(z)=\alpha+(\Delta \alpha) \cos \left(\frac{2 \pi z}{\Lambda}+\Omega\right)(2)
\end{aligned}
$$

여기에서 $n(z)$ 는 굴절률이고, $\alpha(z)$ 는 이득이

다. $z$ 는 장축 방향으로의 좌표이고, $-\frac{L^{\prime}}{2}$ 에서
$\frac{L^{\prime \prime}}{2}$ 까지 변화한다.

만족시켜야 하는 파동 방정식은 다음과 같다.

$$
\nabla^{2} E(z, t)+k^{2}(z) E(z, t)=0
$$

식 (3)의 해는 $E(z, t)=E(z) e^{j \omega t}$ 의 형태로 나 타낼 수 있으며, 다음과 같이 쓸 수 있다.

$k^{2}(z) \approx \beta^{2}+j 2 \alpha \beta+4 \kappa \beta \cos \left(2 \beta_{o} z+\Omega\right)$ (4)

여기서

$$
\begin{gathered}
\beta_{o}=\frac{\pi}{\Lambda} \\
\beta=\frac{\omega}{c} \\
\kappa=\left(\frac{\beta}{2}\right)\left(\frac{\Delta n}{n}\right)+j \frac{\Delta \alpha}{2}
\end{gathered}
$$

식 (4)의 $\alpha$ 는 레이저 발진을 위한 문턱에서의 순 이득이며, 식 (6)의 발진 주파수 $\omega$ 는 $\beta$ 를 알 면 구할 수 있다.

일반성을 유시하년서 $E(z)$ 를 나음과 샅이 쓸 수 있다.

$$
E(z)=R(z) e^{-j \beta_{0} z}+S(z) e^{j \beta_{o} z}
$$

여기서 $R(z)$ 는 양의 $z$ 방향으로 전파하는 파 이고, $S(z)$ 는 음의 $z$ 방향으로 전파하는 파이다. 식 (8)과 (3)으로부터 다음 식을 얻는다.

$$
\begin{gathered}
e^{-j \beta_{o} z}\left[-j 2 \beta_{o} R^{\prime}-\beta_{o}^{2} R+\left(\beta^{2}+j 2 \alpha \beta\right) R\right] \\
+e^{j \beta_{o} z}\left[j 2 \beta_{o} S^{\prime}-\beta_{o}^{2} S+\left(\beta^{2}+j 2 \alpha \beta\right) S\right] \\
+2 \kappa \beta\left(e^{j 2 \beta_{o} z} e^{j \Omega}+e^{-j 2 \beta_{o} z} e^{-j \Omega}\right) \\
\times\left(R e^{-j \beta_{o} z}+S e^{j \beta_{o} z}\right)
\end{gathered}
$$

식 (9)는 다음과 같이 표현할 수 있다.

$$
\begin{aligned}
& -R^{\prime}+(\alpha-j \delta) R=j \kappa e^{-j \Omega} S \\
& S^{\prime}+(\alpha-j \delta) S=j \kappa e^{j \Omega} R
\end{aligned}
$$

여기서 $\delta=\beta-\beta_{o}$ 이고, $|\delta| \ll \beta$ 를 가정하였 고, $e^{ \pm j 3 \beta_{o} z}$ 롤 전파하는 파는 생략하였다.

식 (10)과 (11)은 다음 식의 해를 깆는다.

$$
R(z)=R_{1} e^{\gamma z}+R_{2} e^{-\gamma z}
$$




$$
S(z)=S_{1} e^{\gamma z}+S_{2} e^{-\gamma z}
$$

여기서 $R_{1}, R_{2}, S_{1}, S_{2}$ 는 상수이다.

식 (12)와 (13)을 식 (10)과 (11)에 대입하면 다 음 식을 얻는다.

$$
\begin{aligned}
& \hat{\Gamma} R_{1}=j \kappa e^{-j \Omega} S_{1} \\
& \Gamma R_{2}=j \kappa e^{-j \Omega} S_{2} \\
& \Gamma S_{1}=j \kappa e^{j \Omega} R_{1} \\
& \widehat{\Gamma} S_{2}=j \kappa e^{j \Omega} R_{2}
\end{aligned}
$$

여기서

$$
\begin{aligned}
& \widehat{\Gamma}=-\gamma+\alpha-j \delta \\
& \Gamma=\gamma+\alpha-j \delta
\end{aligned}
$$

식 (14) (17)은 다음 식이 만족될 때 해를 가진 다.

$$
\gamma^{2}=(\alpha-j \beta)^{2}+\kappa^{2}
$$

식 (12)와 (13)으로 표현되는 $R(z)$ 와 $S(z)$ 는 왼쪽 거울 면이 위치한 $z=-\frac{L^{\prime}}{2}$ 과 오른쪽 거울 면이 위치한 $z=\frac{L^{\prime \prime}}{2}$ 에서 다음의 경계 조건을 만족해야 한다.

$$
\begin{aligned}
& e^{j \beta_{o} L^{\prime} / 2}\left(R_{1} e^{-\gamma L^{\prime} / 2}+R_{2} e^{\gamma L^{\prime} / 2}\right) \\
& =\hat{\rho}_{l} e^{-j \beta_{o} L^{\prime} / 2}\left(S_{1} e^{-\gamma L^{\prime} / 2}+S_{2} e^{\gamma L^{\prime} / 2}\right) \\
& e^{j \beta_{o} L^{\prime \prime} / 2}\left(S_{1} e^{\gamma L^{\prime \prime} / 2}+S_{2} e^{-\gamma L^{\prime \prime} / 2}\right) \\
& =\hat{\rho}_{r} e^{-j \beta_{o} L^{\prime \prime} / 2}\left(R_{1} e^{\gamma L^{\prime \prime} / 2}+R_{2} e^{-\gamma L^{\prime \prime} / 2}\right)
\end{aligned}
$$

$\hat{\rho}_{l}$ 과 $\hat{\rho}_{r}$ 은 각각 왼쪽과 오른쪽 거울 면에서의 $E(z)$ 의 반사계수이다. 식 (15) (18)과 (21), (22) 로부터 다음 식이 유도된다.

$$
\frac{e^{-\gamma\left(L^{\prime}+L^{\prime \prime}\right) / 2}\left(1-\rho_{l} \frac{\widehat{\Gamma}}{j \kappa}\right)}{\rho_{l}-\frac{\widehat{\Gamma}}{j \kappa}}
$$

$$
=\frac{e^{\gamma\left(L^{\prime}+L^{\prime \prime}\right) / 2}\left(\rho_{r}-\frac{\widehat{\Gamma}}{j \kappa}\right)}{1-\rho_{r} \frac{\widehat{\Gamma}}{j \kappa}}
$$

여기서

$$
\begin{aligned}
& \rho_{l}=\hat{\rho}_{l} e^{-j \beta_{o} L^{\prime}} e^{j \Omega} \\
& \rho_{r}=\hat{\rho}_{r} e^{-j \beta_{o} L^{\prime \prime}} e^{-j \Omega}
\end{aligned}
$$

식 (14), (16), (18), (19)로부터 다음 식이 유도 된다.

$$
\gamma=\frac{-j \kappa}{2}\left(\frac{\widehat{\Gamma}}{j \kappa}-\frac{j \kappa}{\widehat{\Gamma}}\right)
$$

식 (23)과 (26)으로부터 다음의 고유치 방정식 을 얻는다.

$$
\begin{aligned}
& \gamma L=\frac{-j \kappa L \sinh (\gamma L)}{D} \\
& \times\left(\rho_{l}+\rho_{r}\right)\left(1-\rho^{2}\right) \cosh (\gamma L) \\
& \pm \frac{-j \kappa L \sinh (\gamma L)}{D}\left(1+\rho^{2}\right) \\
& \times\left[\left(\rho_{l}-\rho_{r}\right)^{2} \sinh ^{2}(\gamma L)+\left(1-\rho^{2}\right)^{2}\right]^{1 / 2} \\
& \text { 여기서 } \\
& \quad D=\left(1+\rho^{2}\right)^{2}-4 \rho^{2} \cosh ^{2}(\gamma L) \\
& \quad \rho^{2}=\rho_{l} \rho_{r}=\hat{\rho}_{l} \hat{\rho}_{r} e^{-j 2 \beta_{o} L} \\
& \quad L=\left(L^{\prime}+L^{\prime \prime}\right) / 2
\end{aligned}
$$

식 (27)에서 $\gamma$ 를 구할 수 있으며, 이로부터 문 턱에서의 이득 $\alpha$ 와 $\delta$ 를 구하고, $\delta$ 로부터 발진 주파수 $\omega=\frac{c \beta}{n}=\frac{c}{n}\left(\beta_{o}+\delta\right)$ 를 구할 수 있다.

\section{2 종 방향 빔 분포}

$z$ 방향으로 빔 분포 변화를 다음과 같이 계산 하였다.

식 (12)와 (13)의 $R(z)$ 와 $S(z)$ 표현식에서 $R_{1}, R_{2}, S_{1}, S_{2}$ 의 싱대적 크기를 알 수 있다. 식 (14)로부터 


$$
R_{1}=\frac{j \kappa e^{-j \Omega} S_{1}}{\widehat{\Gamma}}
$$

식 (21), (17), (14)로부터

$$
R_{2}=\frac{1-\rho_{l} \frac{\widehat{\Gamma}}{. j \kappa}}{\rho_{l}-\frac{\widehat{\Gamma}}{j \kappa}} e^{-j \Omega} e^{-\gamma L^{\prime}} S_{1}
$$

식 (17)과 (32)로부터

$$
S_{2}=\frac{j \kappa}{\widehat{\Gamma}} \frac{1-\rho_{l} \frac{\widehat{\Gamma}}{j \kappa}}{\rho_{l}-\frac{\widehat{\Gamma}}{j \kappa}} e^{-\gamma L^{\prime}} S_{1}
$$

$\mathrm{DFB}$ 레이저 내 임의의 $z$ 값에서 $R(z)$ 로 표 현되는 파의 전력은 다음과 같이 구할 수 있다.

$$
P_{a}(z)=c\left|R_{1} e^{\gamma z}+R_{2} e^{-\gamma z}\right|^{2}
$$

마찬가지로 $S(z)$ 로 표현되는 파의 전력은 다 음과 같다.

$$
P_{b}(z)=c\left|S_{1} e^{\gamma z}+S_{2} e^{-\gamma z}\right|^{2}
$$

여기서 $c$ 는 상수이다. 따라서 식 (31), (32), (33)을 식 (34), (35)에 대입하면, $P_{a}(z)$ 및 $P_{b}(z)$ 의 분포를 구할 수 있다. 마찬가지로 식 (31), (32), (33)을 식 (12), (13)에 대입하면 $R(z)$ 와 $S(z)$ 의 분포를 구할 수 있다.

왼쪽 거울 면에서 방사되는 전력 $P_{l}$ 및 우측 거울 면에서 방사되는 전력 $P_{r}$ 은 다음과 같다.

$$
\begin{aligned}
& P_{l}=\left(1-\left|\rho_{l}\right|^{2}\right) P_{b}\left(\frac{-L^{\prime}}{2}\right) \\
& P_{r}=\left(1-\left|\rho_{r}\right|^{2}\right) P_{a}\left(\frac{L^{\prime \prime}}{2}\right)
\end{aligned}
$$

이상과 같은 계산을 $\left(\rho_{l}\right.$ 의 위상 $=0, \rho_{r}$ 의 위상 $=0)$ 인 경우와 $\left(\rho_{l}\right.$ 의 위상 $=0, \rho_{r}$ 의 위상 $\left.=\pi\right)$ 인 경 우에 대하여 서로 비교하였다. 각각에 대하여 $\delta L>0$ 인 경우와 $\delta L<0$ 인 경우로 나누어, 문 턱에서 최소 이득을 필요로 하는 제 1 모드에 대 하여, 빔의 분포 $|R(z)|$ 와 $|S(z)|$, 그리고 방사
전력비 $P_{l} / P_{r}$ 를 구했다.

\section{3. 시뮬레이션 결과}

$3.1 \rho_{l}$ 의 위상 $=0, \rho_{r}$ 의 위상 $=0$

그림 1 은 $\kappa L$ 이 0.1 에서 10 까지 증가할 때 $(\alpha L, \delta L)$ 의 변화를 보여주는 그래프이다.

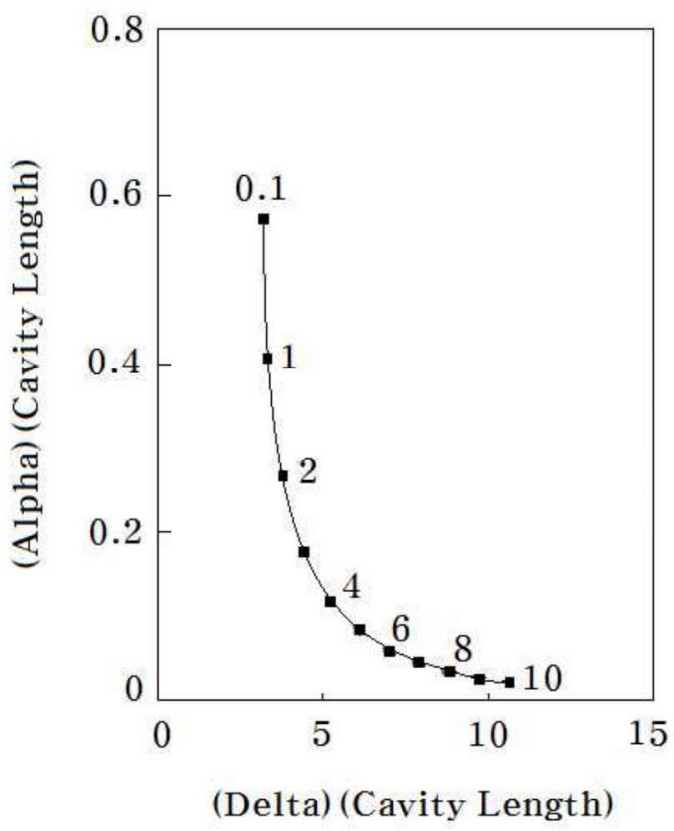

그림 1. $\rho_{l}$ 의 위상 $=0, \rho_{r}$ 의 위상=0인 경우, $\kappa L$ 이 증가할 때 $(\alpha L, \delta L)$ 의 변화 그래프

Fig. 1. The graph of $(\alpha L, \delta L)$ in case of $\rho_{l}$ phase $=0$ and $\rho_{r}$ phase $=0$, as $\kappa L$ increases

그림 2는 $\kappa L=0.1, \kappa L=1.0, \kappa L=10$ 인 세 경우에 대하여 $|R(z)|$ 와 $|S(z)|$ 를 임의의 크기 로 하여 그린 것이다.

그림에서 알 수 있는 바와 같이 $\kappa L=0.1$ 인 
경우 격자의 효과는 거의 나타나지 않는다. $R(z) \sim R_{1} e^{\gamma z}$ 이고 $S(z) \sim S_{2} e^{-\gamma z}$ 인 변화를 보 여준다.

$\kappa L=10$ 인 경우 격자의 효과가 두드러져, 거 울 면보다는 격자에 의해 반사가 일어남을 보여 주고 있다.

$\kappa L$ 에 관계없이 $P_{l} / P_{r}=1$ 임을 알 수 있다.

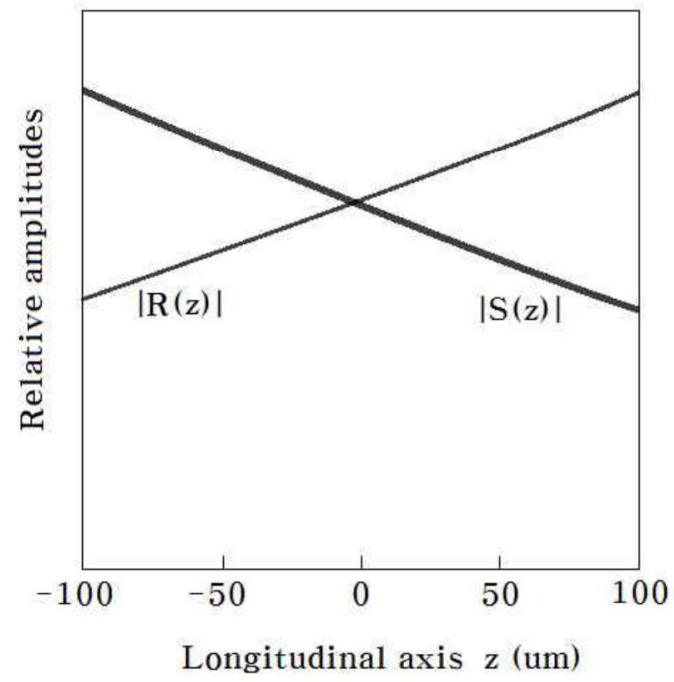

(a) $\kappa L=0.1$

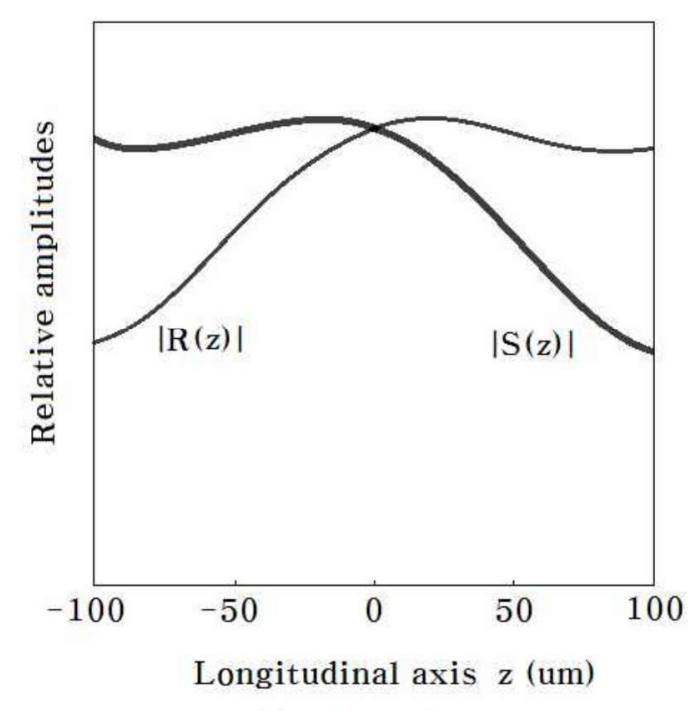

$3.2 \rho_{l}$ 의 위상 $=0, \rho_{r}$ 의 위상 $=\pi$

그림 3 은 $\kappa L$ 이 0.1 에서 10 까지 증가할 때 $(\alpha L, \delta L)$ 의 변화를 보여주는 그래프이다.

그림 4 를 보면, $\kappa L=0.1$ 인 경우 격자의 효과 는 거의 나타나지 않고 지수함수형 증가를 보여 준나. 즉 $R(z) \sim R_{1} e^{\gamma z}$ 이고 $S(z) \sim S_{2} e^{-\gamma z}$ 인 변 화를 보여준다.

$\kappa L=1.0$ 및 $\kappa L=10$ 으로 증가하면서 격자 에 의한 반사가 증가함을 알 수 있다. 그러나 그 림 2 의 경우와는 달리 빔 분포가 $z=0$ 을 중심 으로 비대칭인 것이 특징이며, 이는 거울 면의 위상으로 인한 결과이다.

(b) $\kappa L=1.0$ 


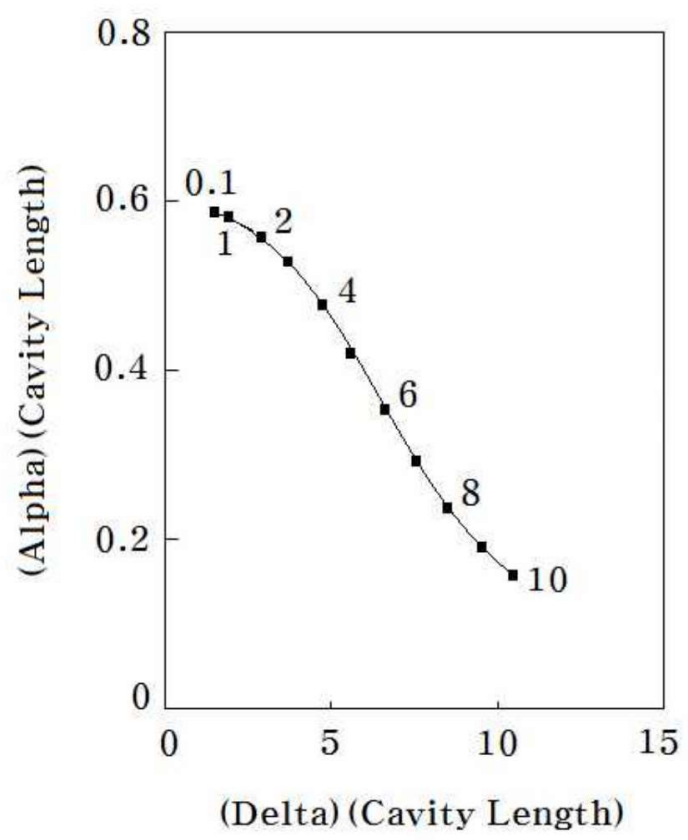

그림 3. $\rho_{l}$ 의 위상 $=0, \rho_{r}$ 의 위상 $=\pi$ 인 경우, $\kappa L$ 이 증가할 때 $(\alpha L, \delta L)$ 의 변화 그래프

Fig. 3. The graph of $(\alpha L, \delta L)$ in case of $\rho_{l}$ phase $=0$ and $\rho_{r}$ phase $=\pi$, as $\kappa L$ increases

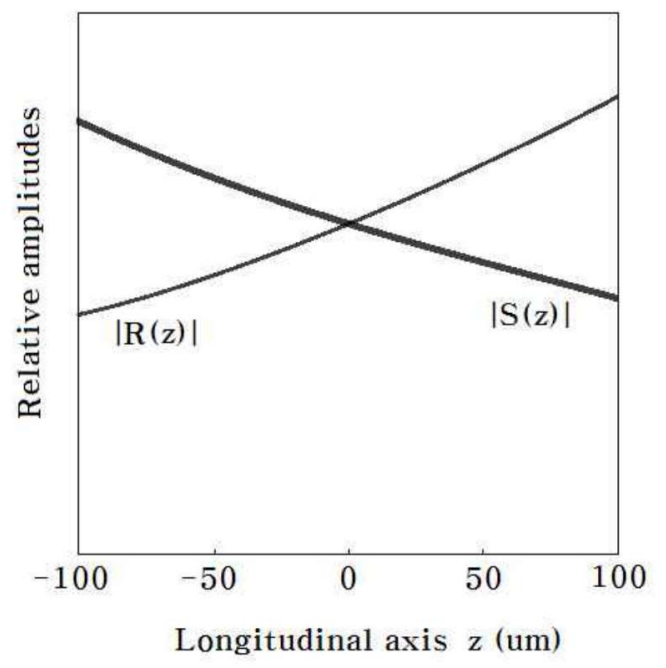

(a) $\kappa L=0.1$

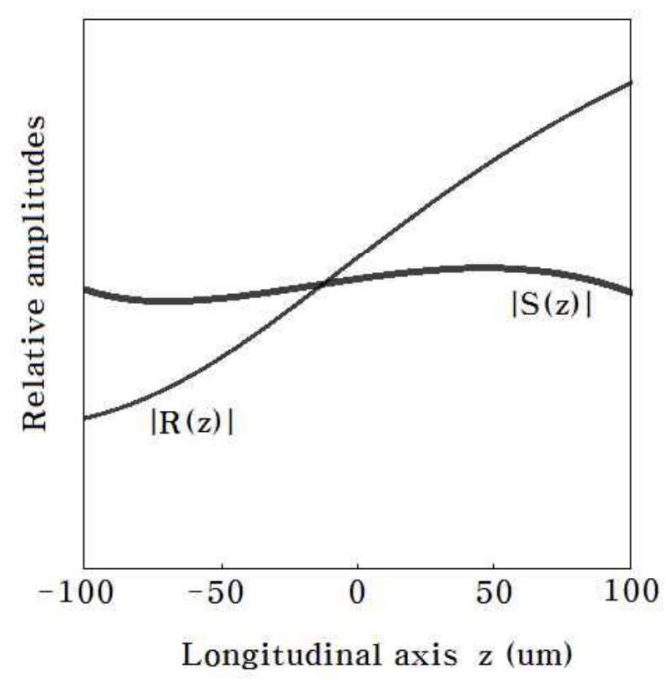

(b) $\kappa L=1.0$

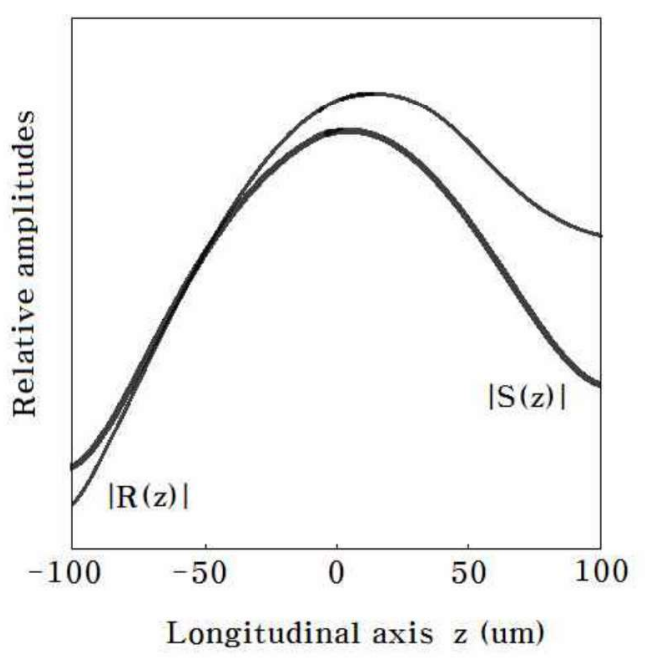

(c) $\kappa L=10$

그림 4. $\rho_{l}$ 의 위상 $=0, \rho_{r}$ 의 위상 $=\pi$ 인 경우, 발진 모드의 빔 분포 $|R(z)|$ 및 $|S(z)|$. (a) $\kappa L=0.1$, (b) $\kappa L=1.0$, (c) $\kappa L=10$

Fig. 4. The beam profiles of $|R(z)|$ and $|S(z)|$ of a lasing mode in case of $\rho_{l}$ phase $=0$ and $\rho_{r} \quad$ phase $=\pi$. (a) $\kappa L=0.1$, (b) $\kappa L=1.0$ and (c) $\kappa L=10$ 
$P_{l} / P_{r}$ 이 더 이상 1 이 아니고, 표 1 과 같이 변 화한다.

표 1. $\kappa L$ 에 따른 $P_{l} / P_{r}$ 의 값 Table 1 . The value of $P_{l} / P_{r}$ as $\kappa L$ increases

\begin{tabular}{|c|c|c|c|}
\hline$\kappa L$ & 0.1 & 1.0 & 10 \\
\hline$P_{l} / P_{r}$ & 0.88 & 0.30 & 0.064 \\
\hline
\end{tabular}

\section{4. 결 론}

무반사 코팅을 하지 않은, 두 거울 면을 가진 $1.55 \mathrm{um}$ 의 파장을 갖는 $\mathrm{DFB}$ 레이저에서 이득 격 자와 굴절률 격자가 동시에 존재할 때, 이득 격 자와 굴절률 격자 사이의 구조와 배치 방법에 따 라 발진 주파수와 발진 이득, 종 방향으로의 빔 분포 특성을 이론적으로 해석한다. 굴절률 격자 와 이득 격자가 거울 면에서 갖는 위상 값의 변 화에 따라서, 그리고 $\kappa L=0.1, \kappa L=1.0$ 및 $\kappa L=10$ 으로 증가하면서, $\mathrm{DFB}$ 레이저의 발진 모드에 대한 빔의 분포 $|R(z)|$ 와 $|S(z)|$, 그리 고 방사전력비 $P_{l} / P_{r}$ 를 구했다. 편의상 다음의 두 가지 경우에 대한 특성을 비교 검증하였다.

(1) $\rho_{l}$ 의 위상 $=0, \rho_{r}$ 의 위상 $=0$

(2) $\rho_{l}$ 의 위상 $=0, \rho_{r}$ 의 위상 $=\pi$

$\rho_{l}$ 의 위상 $=0, \rho_{r}$ 의 위상 $=0$ 인 경우 $\kappa L=1.0$ 이 될 때부터 격자에 의한 반사 효과가 드러나기 시 작함을 볼 수 있고, $\kappa L=10$ 에 이를 때에는 그 림 2(c)에서 보듯이 반사 효과가 매우 두드러지 게 나타남을 볼 수 있다. $\rho_{r}$ 의 위상이 $\pi$ 로 증가 하면, 그림 $4(\mathrm{~b})$ 에서 보듯이 이 반사 효과는 다소 저하되는 것을 볼 수 있다. 따라서 그림 3 에서
보듯이 발진 모드의 문턱 이득도 증가하게 된다. $\rho_{r}$ 의 위상이 0 일 때보다 $\rho_{r}$ 의 위상이 $\pi$ 로 증가 하면서, $\kappa L=2$ 에서부터 $\kappa L=6$ 의 범위에서 가 장 발진 모드의 문턱 이득 증가가 크다는 것을 알 수 있다. 격자에 의한 반사 효과를 두드러지 게 하여 발진 주파수의 안정성을 높이고, 발진 모드의 문턱 전류를 낮추기 위해서는, $\kappa L$ 을 8 보 다 크게 하는 것이 좋다. 또한 $\rho_{r}$ 의 위상 제어가 어려운 상황에서 $\rho_{r}$ 의 위상에 관계없이 낮은 문 턱 전류를 얻기 위해서도, $\kappa L$ 을 8 보다 크게 하 는 것이 비람직히디.

\section{참 고 문 헌}

[1] T.L. Koch and U. Koren, "Semiconductor lasers for coherent optical fiber communications", Lightwave Technology Journal of, Vol. 8, No. 3, pp.274-293, 1990. DOI: $10.1109 / 50.50725$

[2] H. Olesen, J. Salzman, B. Jonsson, and B. Tromborg, "Single-mode stability of DFB lasers with longitudinal Bragg detuning", IEEE Photonics Technology Letters, Vol. 7, Issue 5, pp.461-463, 1995. DOI: $10.1109 / 68.384510$

[3] S.K.B. Lo, and H. Ghafouri-Shiraz, "A method to determine the above-threshold stability of distributed feedback semiconductor laser diodes", Journal of Lightwave Technology, Vol. 13, No. 4, pp.563-568, 1995. DOI: 10.1109/50.372466

[4] C.A. Ferreira Fernandes, "Stability in single longitudinal mode operation in DFB laser structures", Electrotechnical Conference 2004. MELECON 2004. Proceedings of the 12th IEEE Mediterranean, Vol. 1, pp.3-6, 2004. DOI: 10.1109/MELCON.2004.1346756

[5] Jing-Yi Wang, M. Cada, and Jin Sun, 
"Theory for optimum design and analysis of distributed-feedback lasers", IEEE Photonics Technology Letters, Vol. 11, Issue 1, pp.24-26, 1999. DOI: $10.1109 / 68.736378$

[6] M. Okai, S. Tsuji, N. Chinone, "Stability of the longitudinal mode in lambda/4-shifted InGaAsP/InP DFB lasers", IEEE Journal of Quantum Electronics, Vol. 25, Issue 6, pp.1314-1319, 1989. DOI: 10.1109/3.29262

[7] T. Yamanaka, S. Seki, and K. Yokoyama, "Numerical analysis of static wavelength shift for DFB lasers with longitudinal mode spatial hole burning", IEEE Photonics Technology Letters, Vol. 3, Issue 7, pp.610-612, 1991. DOI: $10.1109 / 68.87929$

[8] G. Morthier and R. Baets, "Design of index-coupled DEB lasers with reduced longitudinal spatial hole burning", Journal of Lightwave Technolugy, Vol. 9, No. 10, pp.1305-1313, 1991. DOI: 10.1109/50.90928

[9] T.K. Sudoh, Y. Nakano, K. Tada, K. Kikuchi, T. Hirata, and H. Hosomatsu, "Self-suppression effect of longitudinal spatial hole burning in absorptive-grating gain-coupled DFB lasers", Photonics Technology Letters IEEE, Vol. 5, No. 11, pp.1276-1278, 1993. DOI: 10.1109/68.250043

[10] X. Pan, H. Olesen, and B. Tromborg, "Spectral linewidth of DFB lasers including the effects of spatial hole-burning and nonuniform current injection", Photonics Technology Letters IEEE, Vol. 2, No. 5, pp.312-315, 1990. DOI: $10.1109 / 68.54690$

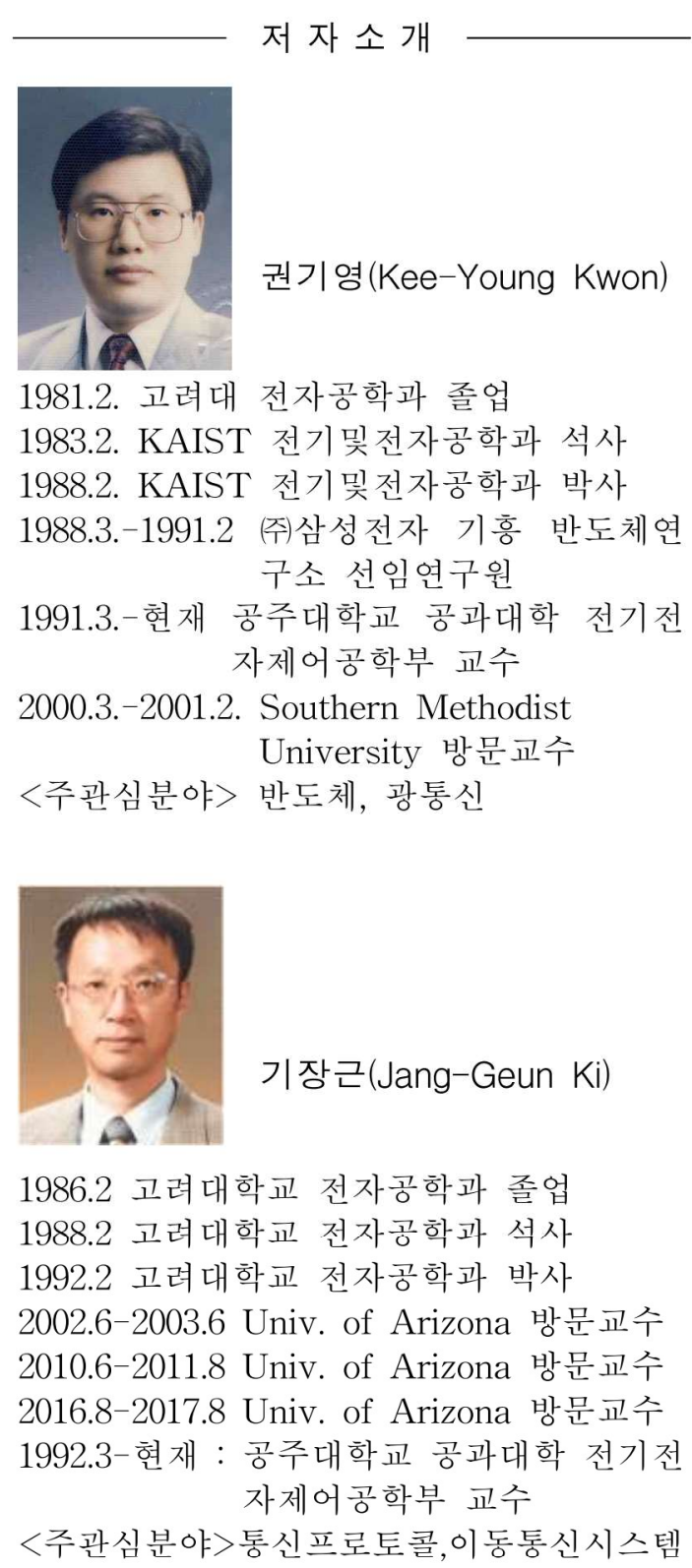

<주관심분야>통신프로토콜,이동통신시스템 